\title{
Perioperative performance of a new Pelvic Organ Prolapse Suspension (POPS) for vaginal vault prolapse
}

\author{
Lucio MA Cipullo ${ }^{*}$, Gianmario E Poto ${ }^{1}$, Slobodan Milosavljevic ${ }^{2}$, Vincenzo Altieri ${ }^{3}$ and Maurizio Guida ${ }^{4}$ \\ ${ }^{1}$ Department of Gynaecology and Obstetrics, University of Salerno, Salerno, Italy \\ ${ }^{2}$ VU University Medical Center Amsterdam, The Netherlands \\ ${ }^{3}$ Department of Urology, University of Salerno, Salerno, Italy \\ ${ }^{4}$ Department of Obstetrics and Gynaecology, University of Naples 'Federico II', Naples, Italy
}

\begin{abstract}
Study objective: To show the results of a new laparoscopic procedure for suspension of the vaginal vault.

Methods: Forty-two previously hysterectomised women underwent the procedure between March 2016 and December 2018 . Prolapse was preoperatively evaluated with the Pelvic Organ Prolapse Quantification (POPQ) score. Women's symptoms and prolapse conditions were re-evaluated with POPQ score after the surgical procedure and at 1-year-follow-up.

Results: Preoperatively, 88.1\% (37/42) of women had a POPQ stage $\geq 2$. Postoperatively, 57.1\% (24/42) showed a POPQ stage of 0 and 38.1\% (16/42) a POPQ stage of 1 , while 2 patients (4.7\%) were lost to Follow-Up (FU). After 1 year, only 15\% of women having surgery (6/40) had a POPQ-stage of 1 and $85 \%$ (34/40) showed no signs of prolapse (POPQ stage 0$)$.

Conclusion: In our work, Laparoscopic Pelvic Organ Prolapse Suspension (LPOPS) showed a high rate of anatomical and functional success. The technique is easy to perform when compared to traditional treatments, offering a relevant reduction of the Pelvic Organ Prolapse (POP) and the complete disappearance of the preoperative symptomatology in most women. Results are even better after 1 year from the procedure.
\end{abstract}

\section{Introduction}

Pelvic Organ Prolapse (POP) is a relentless increase condition, impacting on quality of life when affecting physical performances, emotional reactions and sleep status, giving pain or conditioning social isolation. Lifetime risk of undergoing surgery for a pelvic floor disorder is around $11 \%$, with a reoperation rate for recurrence of $29,2 \%$ [1]. Increase in the average age of the female population, pregnancy, childbirth, obesity and genetics have all been cited as aetiological factors [2].

Vaginal vault prolapse is defined as descensus of the vaginal cuff below $2 \mathrm{~cm}$ less than the total vaginal length above the plane of the hymen [3]. The most common clinical presentation is a bulge or protrusion through the vagina associated with many degrees of coexisting bladder disfunctions, such as frequency, urgency, Stress Urinary Incontinence (SUI) and Urge Urinary Incontinence (UUI) [4]. Way of previous hysterectomy (abdominal or vaginal) doesn't influence the occurrence of the disorder [5]. Management of vaginal vault prolapse depends on the age at presentation, comorbidity (which may impact on the choice of surgery), desire to preserve sexual function and to obtain satisfactory aesthetic outcomes.

The adoption of minimally invasive surgery is exponentially growing, also due to the expected increased demand from women for correction surgeries of UI and POP over the next 40 years [6]. Good conditions of the pelvic floor tone and of the endopelvic fascia are functional to a vaginal procedure (sacrospinous fixation and vaginal mesh procedures), but a high rate of sexual dysfunction and mesh erosion is described, in case of long-term follow-up. Moreover, on April 2019, FDA (Food and Drug Administration) ordered to stop selling surgical meshes intended for transvaginal repair of anterior compartment prolapse (cystocele), because they failed in demonstrating themselves reasonably safe and effective [7]. On the other hand, abdominal and laparoscopic sacrocolpopexy, while improving sexual function, avoiding dyspareunia, need longer hospital stay and time for recovery and mainly show a higher rate of complications [4].

Robotic surgery principles resemble the laparoscopy ones, with the advantage of increased instrument dexterity and 3-dimensional vision. The surgical results are similar, but costs of robotics are extraordinarily more elevated.

In this scenario, we applied this innovative surgical technique, with the aim of demonstrating its effectiveness.

\section{Material and methods}

We enrolled 42 previously hysterectomised women with symptomatic POP. Medical history was obtained regarding age, Body Mass Index (BMI), parity, hypertension, smoking, diabetes. Patients were divided in four groups according to the type of previous

${ }^{\star}$ Correspondence to: Lucio MA Cipullo Department of Gynaecology and Obstetrics, University of Salerno, Salerno, Italy, E-mail: luciocipullo@hotmail.com

Key words: pelvic organ prolapse, laparoscopy, hysterectomy, POPQ score, mesh

Received: March 21, 2020; Accepted: April 24, 2020; Published: April 29, 2020 
hysterectomy: Abdominal Hysterectomy (AH), Vaginal Hysterectomy (VH), Laparoscopy Assisted Vaginal Hysterectomy (LAVH), Total Laparoscopic Hysterectomy (TLH).

The presence of SUI and UUI were investigated with specific questions of a non- standardized questionnaire. Faecal incontinence and other faecal disorders were also investigated. The same interview about urinary and faecal incontinence was performed after the surgical procedure.

The pelvic prolapse was staged by using the Pelvic Organ Prolapse Quantification score (POPQ), described by the International Continence Society [8], before and after the surgical procedure.

\section{Laparoscopic surgical technique}

A standard prophylactic coverage was offered to the patients (Cephalosporin $2 \mathrm{~g}$ "one shot"). General anaesthesia was necessary in all cases. The patient was placed in the lithotomy position and a Foley catheter was inserted. Equipe position was the following: surgeon on the left side of the patient, first assistant on the right side of the patient, second assistant between patient's legs. The pneumoperitoneum was established via umbilical open technique, and a $30^{\circ}$ optical scope was inserted. One $5 \mathrm{~mm}$ trocar was inserted under vision into the point of intersection between umbilical-transverse line and mammary line in the right side and another $5 \mathrm{~mm}$ trocar was inserted symmetrically in the left side. Another $5 \mathrm{~mm}$ trocar was inserted in a suprapubic position.

The procedure included the following steps:

1. Peritoneal cavity exploration and patient in Trendelenburg ( $30^{\circ} \mathrm{C}$ degrees).

2. A vaginal valve was pushed up to the anterior fornix and an intravaginal pad was placed to offer an appropriate exposure of the posterior vaginal fornix and Douglas space

3. A V-shaped $25 \mathrm{~cm}$ long and $2 \mathrm{~cm}$ wide strip was prepared, using a Prolene mesh (Johnson and Johnson)

4. A $3-\mathrm{cm}$ incision of the peritoneum, at the apex of the anterior vaginal fornix and two 3 -cm cutaneous incisions were performed 3 $\mathrm{cm}$ below and $3 \mathrm{~cm}$ medially to the lateral trocars. The aponeurosis of the external oblique muscle was incised. Dissociating the fibers of the internal oblique and transverse muscles by scissors, using long forceps, we realized a subperitoneal long tunnel up to the vaginal cuff.

5. The mesh was introduced into the abdominal cavity through a 10 $\mathrm{mm}$ trocar and fixed by a n. 0 Vycril stitch on the vaginal cuff by a n. 0 Vycril stitch (Figure. 1).

6. The tip of the clamp was then forced out from the peritoneal incision previously performed. One end of the V-mesh was then taken out and pulled out through the tunnel (Figure. 2).

7. Following the same steps, the contralateral strip of the mesh was pulled out.

8. Pelvic organ suspension is achieved by making symmetrical tractions on both mesh strips (Figure. 3). A five $\mathrm{cm}$ of excess mesh strip was bilaterally fixed to the muscle fascia by prolene $2 / 0$ stitches.

9. A third operator estimated the grade of mesh traction to ensure the optimal length of the vagina and to avoid overtraction.

10. The skin was sutured by intradermic fashion.

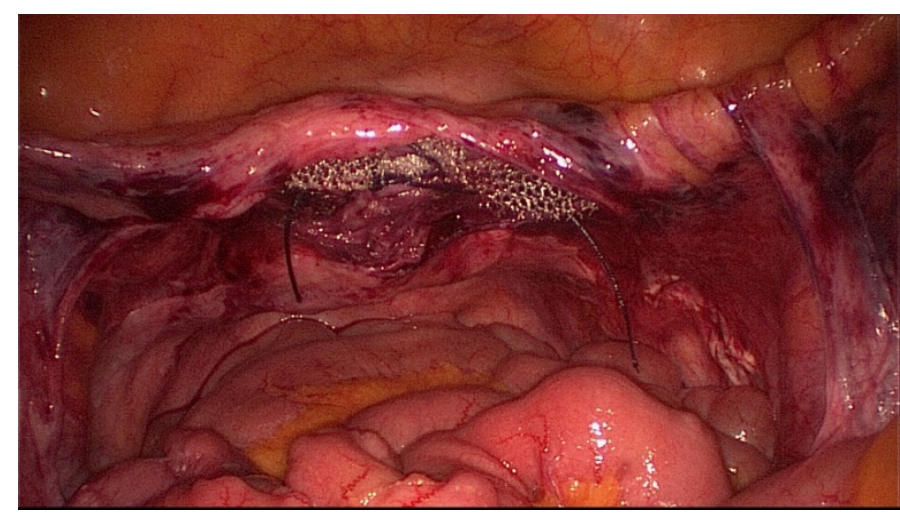

Figure 1. Mesh fixing on the vaginal cuff

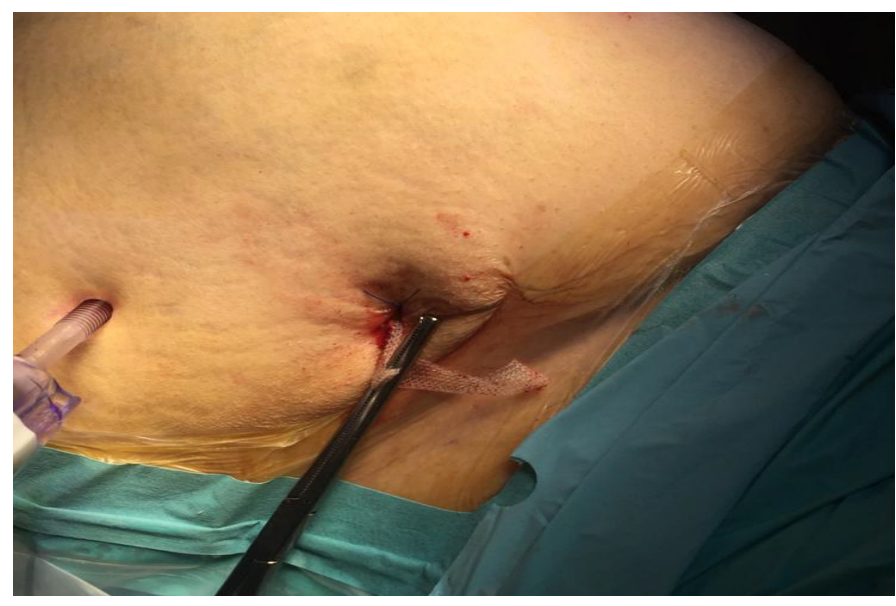

Figure 2. One end of the V-mesh is pulled out through the tunnel

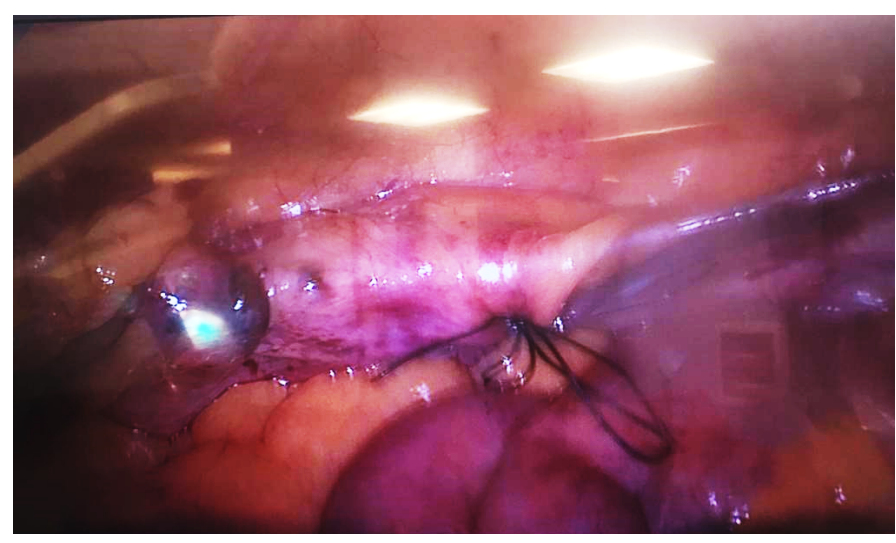

Figure 3. Mesh placed and tightened at the end of surgery

11. The Foley catheter was routinely removed on the 2 nd postoperative day. The patient was discharged three days after surgery, after checking the entity of the eventual Post Void Residual (PVR).

\section{Statistical analysis}

Descriptive statistics were used for the whole population.

To compare POPQ scores and symptoms a paired samples t-test was used. The significance level was set at $\alpha$ of 0.05 and the effect size was calculated by Cohen's d test (which is defined as the difference between two means divided by the pooled standard deviation for those means). An effect size of 0.2 was considered small, 0.5 median and 0.8 or higher as large [9]. 


\section{Results}

Table. 1 shows characteristics of our 42 patients.

The mean age of our pts was 59.8 years and a mean Body Mass Index (BMI) was 27.1.

As said, all 42 patients had previously undergone a hysterectomy: 22 had undergone an $\mathrm{AH}$ (52.4\%), 10 a VH (23.8\%), 7 a TLH (16.7\%), 3 a LAVH (7.1\%). 4 (9.5\%) women had never experience a pregnancy while 28 women $(66.6 \%)$ were multiparous.

2 patients (4.8\%) suffered from faecal incontinence, 3 (7.1\%) from soiling, 15 (35.7\%) from SUI and 6 (14.3\%) from UUI. After surgery, the rate of faecal incontinence and soiling remained identical while remarkable results were achieved on UI: only 1 patient still showed SUI and 5 (11.9\%) UUI, with 1 "de novo" case.

The mean operative time was $75 \pm 15$ minutes. In 2 patients it was impossible to complete the surgical technique because of difficulties on stitching the mesh to the vaginal cuff. One patient suffered of abnormal bleeding, but no intervention required a blood transfusion. 2 patients suffered from post-operative urinary infections.

We measured the rate of success for this surgery by considering the variation of the numeric value of points $\mathrm{Aa}$ and $\mathrm{Ba}$ (anterior vaginal wall), Ap and $\mathrm{Bp}$ (posterior vaginal wall) and $\mathrm{C}$ (vaginal cuff), all reported in Table. 2.

These points describe, quantify and try to stage the level of the pelvic support: they are measured at the level of the vagina correlating with the position of the hymen. Points above the hymen are negative numbers, while points below the hymen are positive numbers. All the measurements are taken at the maximum Valsalva pressure [8]. Our results show that point $\mathrm{C}$ is affected by the greatest variation (mean score: $0.563 \rightarrow-7.712$ ) and the highest effect size (2.55): point $C$ is given by the most distal edge of the vaginal cuff scar: its values best describe surgery results.

Adopting the POPQ as staging criteria, defined by the

Table 1. Characteristics of patients

\begin{tabular}{|c|c|}
\hline Patient characteristics & $\mathrm{n}=\mathbf{4 2}$ \\
\hline Age $^{\mathrm{a}}$ & $59.98(9.09)$ \\
\hline BMI & $27.05(4.62)$ \\
\hline Stress Urinary Incontinence (SUI) ${ }^{b}$ & $15(35.7 \%)$ \\
\hline Urge Incontinence (UI) & $6(14.3 \%)$ \\
\hline Fecal incontinence & $2(4.8 \%)$ \\
\hline Nulliparous & $4(9.5 \%)$ \\
\hline \multicolumn{2}{|l|}{ Previous type of hysterectomy } \\
\hline AH (abdominal hysterectomy) & $22(52.4 \%)$ \\
\hline VH (vaginal hysterectomy) & $10(23.8 \%)$ \\
\hline TLH (total laparoscopic hysterectomy) & $7(16.7 \%)$ \\
\hline LAVH (laparoscopic-assisted vaginal hysterectomy) & $3(7.1 \%)$ \\
\hline Operative time $(\min )^{c}$ & $75(60-90)$ \\
\hline
\end{tabular}

a: Mean (standard deviation); b: Number (\%); c: Range.

Table 2. Values of POPQ staging points before and after surgery

\begin{tabular}{|c|c|c|c|c|}
\hline & Before surgery & After surgery & P-value & Effect size \\
\hline $\mathrm{Aa}$ & 0.025 & -2.725 & $<0.01$ & 1.93 \\
\hline $\mathrm{Ba}$ & 1.15 & -2.625 & $<0.01$ & 1.81 \\
\hline $\mathrm{C}$ & 0.563 & -7.712 & $<0.01$ & 2.55 \\
\hline $\mathrm{Ap}$ & -0.1 & -2.725 & $<0.01$ & 1.63 \\
\hline $\mathrm{Bp}$ & 1.050 & -2.575 & $<0.01$ & 1.75 \\
\hline
\end{tabular}

Aa and Ba: Anterior vaginal wall; Ap and Bp: Posterior vaginal wall; C: Vaginal cuff. overmentioned points, in 40 patients treated with our technique, prolapse was completely corrected in 24 patients (POPQ stage 0 ), obtaining a POPQ stage 1 in the remaining 16 patients.

At a 1-year-follow-up, revaluating patients with POPQ system, results are even better: only $15 \%(6 / 40)$ still showed signs of prolapse with a POPQ stage of 1 , while $85 \%$ of patients $(34 / 40)$ had a complete recovery.

\section{Discussion}

In our study we describe a new laparoscopic procedure of Pelvic Organ Prolapse Suspension and evaluate the preliminary results.

This technique was first described by Ceci, et al. associated with stapled transanal rectal resection (STARR), in women with multiorgan female prolapse [10].

Our study aims to assess effects of this laparoscopic procedure on vaginal vault prolapse after hysterectomy, whose influence on pelvicfloor dysfunction is described in many studies [11]. Atrophy and deficiency of the uterosacral ligaments may loosen the vaginal tension at the bladder base, decreasing the tension of the vaginal membrane and increasing the work of the muscles which favour the outflow during micturition [12].

Mean POPQ stage was respectively 2.18 for the 22 women submitted to $\mathrm{AH}, 2.7$ and 2.66 for those submitted to $\mathrm{VH}$ and TLH. The mean POPQ stage is higher for the other 3 women submitted to LAVH amounting to 3.25 . This is probably due to the different mechanic stress connected to each type of surgery, especially concerning the stretching of the vaginal, cardinal and the other regional ligaments. In addition, other conditions, such as parity, age or BMI could play a major role [13].

Our results confirm that the delivery can contribute in determining the degree of the prolapse. Pregnancy, especially the first, and delivery may increase pelvic organ descent and lower the bladder neck [14]. Vaginal delivery seems to cause the main variation of the vaginal vault position. Among the 20 patients with at least 2 vaginal deliveries, 12 (60\%) showed a POPQ stage $\geq 3$.

Our results show that POPS play a functional and anatomical positive role on vaginal vault prolapse and stress urinary incontinence. Prolapse was completely corrected in 24 patients, obtaining a POPQ score of 1 in 16 patients. In 15 patients presenting SUI before surgery, only 1 was still affected after surgery.

The successful result is achieved by the placement of a sub peritoneal mesh stitched on the vaginal cuff, obtaining the ideal stretching. The procedure also respects the FDA warning [7]: the mesh is located within the sterile environment of the peritoneum. This position also prevents the commonly reported vaginal complications, as mesh erosion and contraction, with vaginal shortening.

In our experience at 1-year-follow-up, this procedure shows a progressive stretching, without loss of tension and no long-term relapses or other complications: $85 \%$ (34/40) of patients get the complete disappearance of prolapse, showing even better results compared with the $60 \%(24 / 40)$ of patients with POPQ stage 0 , evaluated immediately after the procedure.

This technique may be adopted even in case of retained uterus. Preservation of the uterus, suspending it in a natural position, involves significant surgical, functional and psychological benefits. Uterus is an essential symbol of womanhood and hysterectomy can represent a serious psychological trauma, not only affecting the sexual activity. 
On the other side, the technique is obviously affected by risks related to general anaesthesia and laparoscopy (e.g.: pneumothorax, air embolism, subcutaneous emphysema).

The low incidence of complications also contributes to connote this operation as safe and effective mainly for those women who wish to treat such a disorder, obtaining a significant impact on quality of life.

\section{Compliance with ethical standards}

This is an observational study. Campania Sud Research Ethics Committee has confirmed that no ethical approval is required.

This research did not receive any specific grant from funding agencies in the public, commercial or not-for-profit sectors.

Authors declare no conflict of interest.

Informed consent to participate to the study was collected from participants.

\section{References}

1. Olsen AL, Smith VJ, Bergstrom JO, Colling JC, Clark AL (1997) Epidemiology of surgically managed pelvic organ prolapse and urinary incontinence. Obstet Gynecol 89: 501-506.

2. Jones KA, Moalli PA (2010) Pathophysiology of pelvic organ prolapse. Female Pelvic Med Reconstr Surg 16: 79-89.

3. Abrams P, Cardozo L, Fall M, Griffiths D, Rosier P, et al. (2003) The standardisation of terminology of lower urinary tract function: Report from the standardisation subcommittee of the international continence society. Am J Obstet \& Gynecol 187: 116-126.
4. Ramalingam K, Monga A (2013) Management of vault prolapse. Obstet Gynecol 15: $167-170$.

5. De Lancey JO (1992) Anatomic aspects of vaginal eversion after hysterectomy. Am J Obstet Gynecol 166: 1717-1728.

6. Wu JM, Kawasaki A, Hundley AF, Dieter AA, Myers ER, et al. (2011) Predicting the number of women who will undergo incontinence and prolapse surgery, 2010 to 2050. Am J Obstet Gynecol 205: 230.e1-230.e5.

7. https://www.fda.gov/NewsEvents/Newsroom/PressAnnouncements/ucm636114.htm

8. Bump RC, Mattiasson A, Bø K, Brubaker LP, DeLancey JO, et al. (1996) The standardization of terminology of female pelvic organ prolapse and pelvic floor dysfunction. Am J Obstet Gynecol 175: 13.

9. Cohen J (1988) Statistical power analysis for the behavioural sciences, 2nd edition Erlbaum, Hillsdale, NY.

10. Ceci F, Spaziani E, Corelli S, Casciaro G, Martellucci A, et al. (2013) Technique and outcomes about a new laparoscopic procedure: The Pelvic Organ Prolapse Suspension (POPS). G Chir 34: 141-144.

11. Petros PE (2000) Influence of hysterectomy on pelvic-floor dysfunction. Lancet 356: 1275.

12. Petros PE Ulmsten U (1993) An integral theory and its method for the diagnosis and management of female urinary incontinence. ScandJ Urol Nephrol 1993: 1-93.

13. Huei-Kai H, Dah-Ching D (2018) Pelvic organ prolapse surgery following hysterectomy with benign indication: a national cohort study in Taiwan. Int Urogynecol J 29: 16691674.

14. Van Geelen H, Ostergard D, Sand P (2018) A review of the impact of pregnancy and childbirth on pelvic floor function as assessed by objective measurement techniques Int Urogynecol J 29: 327-338.

Copyright: (C2020 Cipullo LMA. This is an open-access article distributed under the terms of the Creative Commons Attribution License, which permits unrestricted use, distribution, and reproduction in any medium, provided the original author and source are credited. 